\title{
Sichere elektronische Identität - ein Ziel für Europa!
}

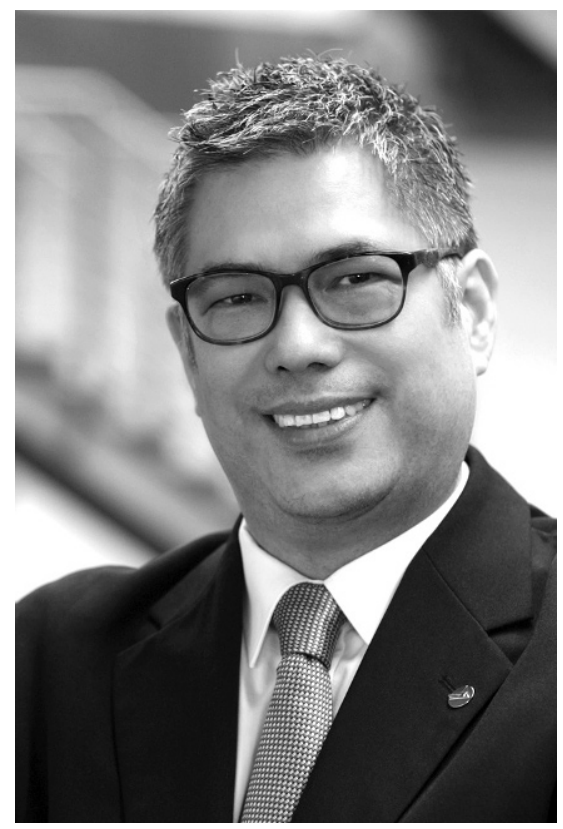

Die Digitalisierung ist wahrscheinlich eine der größten Revolutionen der vergangenen Jahre und Jahrzehnte und hat schon jetzt wesentliche Aspekte unseres täglichen Lebens grundlegend verändert - sei es im privaten oder im beruflichen Umfeld. Die pandemische Lage, in der wir uns nun seit fast zwei Jahren befinden, hat deutlich gemacht, dass eine Digitalisierung von Prozessen im öffentlichen und privaten Sektor eine wesentliche Rolle bei der Aufrechterhaltung der Lebens- und Arbeitsfähigkeit spielt - dabei ist der private Sektor durch Services wie Online-Banking, OnlineShopping etc. deutlich weiter. Die großen globalen Plattformen wie Apple, Google, Facebook \& Co. sind im Bereich User Experience und Convenience sicherlich führend - allerdings sind die rechtlichen Grundlagen und verschiedene Datenschutzaspekte teilweise unklar.

Die Akzeptanz neuer digitaler Prozesse ist im Wesentlichen abhängig von der Vertrauenswürdigkeit der verwendeten Komponenten und Lösungen. Hier spielen sichere Identitäten eine ganz entscheidende Rolle. Die Bundesregierung hat sich dieses Themas schon vor etlichen Jahren angenommen. Ein ganz entscheidender Schritt war die Einführung des Personalausweises mit Online-Ausweisfunktion und dem damit verbundenen Ökosystem im Jahr 2010. Mit der eIDAS-Verordnung der Europäischen Kommission wurde das Thema digitale hoheitliche Identitäten und Vertrauensdienste 2014 dann ein europäisches Thema - mit großer Wirkung nach innen und außen. Nun, sieben Jahren später, ist zu konstatieren, dass die Europäische Union mit der eIDAS-Verordnung einen wesentlichen Schritt hin zu einem einheitlichen digitalen Vertrauensraum gemacht hat. Allerdings haben die Aktivitäten zur Revision der Verordnung, die 2020 gestartet wurden und im Sommer 2021 zu einem ersten Revisionsvorschlag der Kommission geführt haben, wesentlichen Handlungsbedarf aufgezeigt, um eine höhere Akzeptanz und Verbreitung von digitalen Identitäten im europäischen Rahmen zu erreichen.

Auch der im November 2021 veröffentlichte Koalitionsvertrag betont, dass das Potenzial der Digitalisierung in Staat und Gesellschaft besser genutzt werden soll und ein digitaler Aufbruch und eine digitale Souveränität nur im europäischen Rahmen gelingen kann. Gerade für den Ausbau der Digitalisierung der Verwaltung wird ein vertrauenswürdiges, allgemein anwendbares Identitätsmanagement als unabdingbare Basis angesehen, die mit hoher Priorität verfolgt werden soll.

Das Thema der vertrauenswürdigen digitalen Identitäten ist erstmals im Zentrum der technischen und politischen Diskussion sowohl auf nationaler wie europäischer und globaler Ebene angekommen.

Die vorliegende Ausgabe der "Datenschutz und Datensicherheit" widmet sich daher im Detail den verschiedenen Aspekten dieser aktuellen Diskussion auf nationaler und europäischer Ebene

\section{Kim Nguyen}

